\title{
Broadband Cavity Enhanced Differential Optical Absorption Spectroscopy (CE-DOAS) - applicability and corrections
}

\author{
U. Platt ${ }^{1}$, J. Meinen ${ }^{1,2}$, D. Pöhler ${ }^{1}$, and T. Leisner ${ }^{1,2}$ \\ ${ }^{1}$ Inst. for Environmental Physics (IUP), Atmosphere and Remote Sensing, Ruprecht-Karls-Universität Heidelberg, Germany \\ ${ }^{2}$ Institute for Meteorology and Climate Research, Aerosols and Heterogeneous Chemistry in the Atmosphere (IMK-AAF), \\ Forschungszentrum Karlsruhe GmbH, Germany
}

Received: 26 November 2008 - Published in Atmos. Meas. Tech. Discuss.: 23 December 2008

Revised: 20 October 2009 - Accepted: 27 October 2009 - Published: 16 November 2009

\begin{abstract}
Atmospheric trace gas measurements by cavity assisted long-path absorption spectroscopy are an emerging technology. An interesting approach is the combination of CEAS with broadband light sources, the broadband CEAS (BB-CEAS). BB-CEAS lends itself to the application of the DOAS technique to analyse the derived absorption spectra. While the DOAS approach has enormous advantages in terms of sensitivity and specificity of the measurement, an important implication is the reduction of the light path by the trace gas absorption, since cavity losses due to absorption by gases reduce the quality $(\mathrm{Q})$ of the cavity. In fact, at wavelength, where the quality of the BB-CEAS cavity is dominated by the trace gas absorption (especially at very high mirror reflectivity), the average light path will vary nearly inversely with the trace gas concentration and the strength of the band will become only weakly dependent on the trace gas concentration $\mathrm{c}$ in the cavity, (the differential optical density being proportional to the logarithm of the trace gas concentration). Only in the limiting case where the mirror reflectivity determines $\mathrm{Q}$ at all wavelength, the strength of the band as seen by the CE-DOAS instrument becomes directly proportional to the concentration $c$. We investigate these relationships in detail and present methods to correct for the cases between the two above extremes, which are of course the important ones in practice.
\end{abstract}

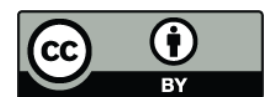

Correspondence to: U. Platt (ulrich.platt@iup.uni-heidelberg.de)

\section{Introduction}

Absorption spectroscopy and in particular Differential Optical Absorption Spectroscopy (DOAS) (e.g. Platt et al., 1979; Platt, 1994; Platt and Stutz, 2008) has been a powerful tool for the measurement of atmospheric trace gases. The technique offers high sensitivity for many gases of interest for atmospheric chemistry (e.g. $\mathrm{NO}_{2}, \mathrm{SO}_{2}, \mathrm{CH}_{2} \mathrm{O}, \mathrm{HONO}$, monocyclic aromatics), inherent calibration, and the capability to measure unstable species (e.g. $\mathrm{NO}_{3}, \mathrm{OH}$, halogen oxide radicals). Typically optical path lengths of several kilometres are required; although multi-reflection optics can be used (see e.g. Platt and Stutz, 2008) they do not usually provide the necessary optical pathlengths, thus preculding insitu DOAS measurements in many applications. On the other hand some scientific applications profit from in-situ measurements. Therefore, combining the advantages of the compact arrangement of long light-paths with the sensitivity and selectivity of DOAS may well prove to become a significant improvement in trace gas measurement technology.

Technologies for gas measurements by cavity assisted long-path absorption spectroscopy are rapidly evolving (e.g. Zalicki and Zare, 1995; Paldus and Zare, 1999; Brown, 2003; Ball et al., 2004), a particular application is the analysis of atmospheric trace gases. Initial applications of cavity-assisted spectroscopy made use of the analysis of the ring-down time of a passive optical resonator at a wavelength at the peak of an absorption line of the trace gas under investigation. Consequently, this technique has become known as Cavity RingDown Spectroscopy (CRDS). CRDS instruments usually use a narrow-band laser as light source (e.g. Brown, 2003).

Alternatively, passive resonators can be used to provide long light paths for absorption spectroscopy analysing the

Published by Copernicus Publications on behalf of the European Geosciences Union. 


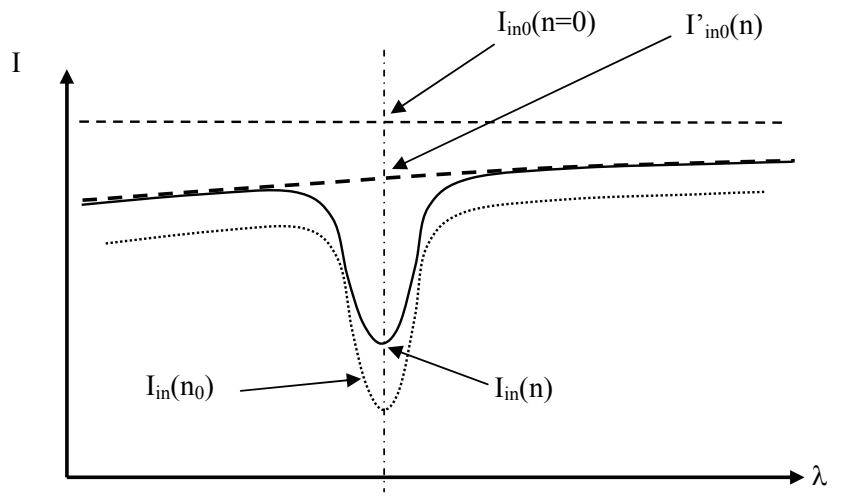

Fig. 1. Reduction of the light path $L_{\mathrm{eff}}$ in a cavity due to extinction in the cavity (expressed as optical density $D_{C E}$ ). Plotted is the ratio $L_{\text {eff }} / L_{0}$, where $L_{0}$ denotes the average light path in an empty (air filled) cavity as described in the text. For illustration the effective light paths at $662 \mathrm{~nm}$ (peak of the $\mathrm{NO}_{3}$ absorption band) for three realistic $\mathrm{NO}_{3}$ total column densities (in $\mathrm{cm}^{-2}$ ) in the cavity are also shown (see Meinen et al., 2009).

transmission of continuous light. This technique has become known as Cavity Enhanced Absorption Spectroscopy (CEAS) (e.g. Engeln et al., 1998; Wheeler et al., 1998; Peeters et al., 2000; Englund, 2002; Fawcett et al., 2002; Brown, 2003; Simpson, 2003; Ball et al., 2004; Fiedler, 2005; Venables et al., 2006; Thompson et al., 2006; Langridge et al., 2006, 2008; Triki et al., 2008; Gherman et al., 2008). An interesting approach is the combination of CEAS with broadband light sources like Xe-arc lamps, LED's, or broadband lasers leading to broadband CEAS (BB-CEAS) (Fiedler et al., 2003; Bitter et al., 2005; Meinen et al., 2008). BB-CEAS lends itself to the application of the DOAS technique for the analysis of the derived absorption spectra.

Several authors applied BB-CEAS DOAS (or CE-DOAS) for the analysis of BB-CEAS - derived wavelength dependent absorption coefficients (Ball et al., 2004; Bitter et al., 2005; Fiedler et al., 2007; Meinen et al., 2008) and also compared results of BB-CEAS measurements to long-path DOAS data. However, to our knowledge there was no systematic study of the implications of applying the DOAS approach to BB-CEAS measurements. Especially the above mentioned reduction of the light path by the trace gas absorption (or aerosol extinction), has not been investigated thoroughly. In the following we investigate these relationships in detail and derive a method to correct for the light path reduction due to trace gas absorption i.e. for the cases between the two above extremes, which are of course the important ones in practice.

\section{The DOAS principle}

The traditional DOAS technique provides the differential optical density (e.g. Platt and Stutz, 2008). This is fre- quently summed up in the equation for the light intensity after traversing an absorption path:

$$
\begin{aligned}
I(\lambda)= & I_{0}(\lambda) \cdot \exp \left[-L \cdot\left(\sum_{j}\left(\sigma_{j}^{\prime}(\lambda) \cdot \bar{c}_{j}\right)\right)\right] \\
& \cdot \exp \left[-L \cdot\left(\sum_{j}\left(\sigma_{j B}(\lambda) \cdot \bar{c}_{j}\right)+\varepsilon_{R}(\lambda)+\varepsilon_{M}(\lambda)\right)\right]
\end{aligned}
$$

Where $I_{0}, I$ refer to the intensities before and after the trace gas absorption has taken place (the $c_{j}$ denote the concentration of the trace gases present in the cavity), respectively, and $\sigma_{B}(\lambda)$ and $\sigma^{\prime}(\lambda)$ denote the broadband and "differential" parts of the cross section, respectively. The first exponential in the above equation describes the differential optical density $D^{\prime}$ due to different trace gases $j$ to be measured, which varies strongly with wavelength. The second exponential describes rather continuous absorption, which varies smoothly with wavelength (see e.g. Platt, 1994, or Platt and Stutz, 2008). The latter is due to Rayleigh scattering by air molecules (extinction coefficient $\varepsilon_{R}$ ), Mie scattering by atmospheric aerosol (extinction coefficient $\varepsilon_{M}$ ) and a possible continuous fraction of the trace gas absorptions. Thus the total trace absorption cross section $\sigma(\lambda)$ is split in a "differential" part $\sigma^{\prime}(\lambda)$ rapidly varying with wavelength (i.e. describing absorption bands or lines) and a rather continuous part $\sigma_{B}(\lambda)$. Accordingly, the optical densities can be written as:

$$
\begin{aligned}
& D=\ln \left[\frac{I_{0}(\lambda)}{I(\lambda)}\right]=L \cdot \sum_{j}\left(\sigma_{j}^{\prime}(\lambda) \cdot \bar{c}_{j}\right)+L \\
& \cdot\left[\sum_{j}\left(\sigma_{j B}(\lambda) \cdot \bar{c}_{j}\right)+\varepsilon_{R}(\lambda)+\varepsilon_{M}(\lambda)\right] \\
& \text { or } \\
& D=D^{\prime}+D_{B} \\
& \text { with } \\
& D^{\prime}=L \cdot \sum_{j}\left(\sigma_{j}^{\prime}(\lambda) \cdot \bar{c}_{j}\right) \text { and } \\
& D_{B}=L \cdot\left[\sum_{j}\left(\sigma_{j B}(\lambda) \cdot \bar{c}_{j}\right)+\varepsilon_{R}(\lambda)+\varepsilon_{M}(\lambda)\right]
\end{aligned}
$$

In DOAS applications, usually not the total optical density is used to determine trace gas concentrations, but rather the differential absorption i.e. its part rapidly varying with wavelength, therefore the optical density $D$ is split in two portions $D_{B}$ and $D^{\prime}$, which are "slowly" and a "rapidly" varying with wavelength (Platt, 1994; Platt and Stutz, 2008). For a single trace gas, we have:

$$
D(\lambda)=\ln \left(\frac{I_{0}(\lambda)}{I(\lambda)}\right)=D_{B}(\lambda)+D^{\prime}(\lambda)=\bar{L} \cdot \bar{c}\left[\sigma_{B}(\lambda)+\sigma^{\prime}(\lambda)\right]
$$

Accordingly, the cavity extinction per unit length is written as:

$$
\alpha=\left[\sigma_{B}(\lambda)+\sigma^{\prime}(\lambda)\right] \cdot \bar{c}+\varepsilon_{B}
$$


where $\varepsilon_{B}$ denotes any other broadband extinction except the Rayleigh extinction measured in the "empty" cavity. The corresponding intensities are illustrated in Fig. 1.

Thus we have:

$$
\begin{aligned}
I(\lambda)= & I_{0}(\lambda) \cdot \exp \left[-L \cdot \sum_{j} \sigma_{j}^{\prime}(\lambda) c_{j}\right. \\
& \left.-L \cdot\left(\sum_{j} \sigma_{0 j}(\lambda) c_{j}+\varepsilon_{R}+\varepsilon_{M}\right)\right]
\end{aligned}
$$

and

$$
\begin{aligned}
D_{\text {meas. }}(\lambda) & =\ln \left(\frac{I_{0}(\lambda)}{I(\lambda)}\right) \\
& =L \cdot \sum_{j} \sigma_{j}^{\prime}(\lambda) c_{j}+L \cdot\left[\sum_{j} \sigma_{0 j}(\lambda) c_{j}+\varepsilon_{R}+\varepsilon_{M}\right]
\end{aligned}
$$

The DOAS evaluation makes use of a modelled spectrum:

$$
D_{\text {Fit }}(\lambda)=\sum_{j} a_{j} \sigma_{j}^{\prime}(\lambda)+\underbrace{\sum_{k} b_{k} \lambda^{k}}_{\text {Polynomial in } \lambda}
$$

determinig the coefficients $a_{j}, b_{k}$ such that the overall square of the deviation between $D_{\text {meas }}$ and $D_{\text {fit }}$ is minimised. In classic long-path DOAS applications the coefficients $b_{k}$ of the polynomial are determined, but not used. However in situations, where the aerosol can be removed from the light path (in order to determine $I_{0}$ ), as in some CE-DOAS instruments, the polynomial can be utilised to obtain the aerosol optical density $D_{\mathrm{Mie}}$ and thus the aerosol extinction $\varepsilon_{M}$ :

$D_{\mathrm{Mie}}(\lambda)=\sum_{k} b_{k} \lambda^{k}-\sum_{j} a_{j} \sigma_{0 j}(\lambda)-\varepsilon_{R}$ and: $\varepsilon_{M}=\frac{D_{\mathrm{Mie}}}{L}$

\section{BroadBand-Cavity Enhanced Absorption Spectrosco- py (BB-CEAS) principles}

The basic idea of CE-DOAS is to introduce incoherent broadband light (intensity $I_{L}$ ) into an optical resonator (see Fig. 2) consisting of two mirrors with reflectivity $R_{1}=R_{2}=R$ (in case of different reflectivities of the two mirrors $R_{1}, R_{2}$ one may take the harmonic average reflectivity $R=\left(R_{1} \cdot R_{2}\right)^{1 / 2}$. Initially only the fraction $\rho=1-R$ of the radiation emitted by the light source, $I_{L}$, will enter the resonator, however, once in the resonator the radiation will be reflected about $1 /(1-R)$ times (neglecting other losses) on average. Finally, in the absence of any extinction in the cavity, half the radiation will leave the resonator through mirror 1 , the other half through mirror 2, each fraction will have the intensity $I_{0}$ :

$I_{0}(\lambda)=I_{L}(\lambda) \cdot \frac{(1-R(\lambda))}{2}=I_{L}(\lambda) \cdot \frac{\rho(\lambda)}{2}$

The wavelength dependence of the variables $I_{0}, I_{L}, R, \rho, \varepsilon_{R}$, $\varepsilon_{B}, L$ is omitted in the following for simplicity.
Since usually "empty cavity" measurements are made with a purified air filled cavity it is convenient to include the Rayleigh extinction $\varepsilon_{R}$ of pure air in an effective mirror reflectivity $R_{0}$ :

$R_{0}=R-\varepsilon_{R} d_{0}$ or $\rho_{0}=1-R_{0}=1-R+\varepsilon_{R} d_{0}=\rho+\varepsilon_{R} d_{0} \cdot(10)$

Where the index 0 always corresponds to the cavity without absorbers. A detailed discussion of the relationship between reflectance, extinction, and differential absorption is given by Fiedler (2005) and Fiedler et al. (2005, 2007).

From the change of one traverse of the resonator we derive for the decay of the intensity of a light pulse inside the cavity $I_{\text {in }}$ :

$$
-\frac{d I_{i n}(n)}{d n}=I_{\text {in }}(n) \cdot(1-T R)
$$

(Note that the number of reflection $n$ is used as a continuous variable here, although in reality $n$ can only assume discrete values).

With:

$T=$ Transmissivity of the cavity (see Fig. 2)

$R=$ Reflectivity of the mirror (see Fig. 2, assumed to be equal for $M_{1}$ and $M_{2}$ )

$n=$ number of light traverses through the cavity

Although in practice $n$ can only assume positive integer values the above equation is derived for any positive value of $n$, it is in particular correct for integer values. Equation (11) can be integrated with the boundary condition that the initial light intensity before the first traverse through the cavity is $I_{\text {in }}(n=0)=I_{\text {in }}(0)$ :

$\ln \left(\frac{I_{\text {in }}(0)}{I_{\text {in }}(n)}\right)=n \cdot(1-T R)$

or:

$I_{\text {in }}(n)=I_{\text {in }}(0) \cdot \exp [n \cdot(T R-1)]$

Where $I_{\text {in }}(n)$ denotes the intensity inside the cavity after $\mathrm{n}$ reflections (or traverses through the cavity). Re-writing $T=1-\tau$, $R=1-\rho$ with $\tau, \rho \ll 1$ (Note: here we use the symbol $\tau=1$ Transmission, not to be confused with the ring-down time or optical density) we obtain:

$$
T R=(1-\tau)(1-\rho)=1-\tau-\rho+\underbrace{\tau \rho}_{\approx 0} \approx 1-\tau-\rho
$$

Introducing $\mathrm{TR}$ from above in the expression for $\ln \left[I_{\text {in }}(0) / I_{\text {in }}(n)\right]$, Eq. (12), and replacing $\rho$ by $\rho_{0}$ (i.e. considering an air-filled cavity, see Eq. 10):

$$
\begin{aligned}
\ln \left(\frac{I_{\text {in }}(0)}{I_{\text {in }}(n)}\right) & =n \cdot(1-T R) \approx n \cdot\left(1-\left(1-\tau-\rho_{0}\right)\right) \\
& =n \cdot\left(\tau+\rho_{0}\right)
\end{aligned}
$$

Note that according to Lambert-Beer's law $\tau$ can be written as:

$$
\tau=\sigma \cdot \bar{c} \cdot d_{0}
$$




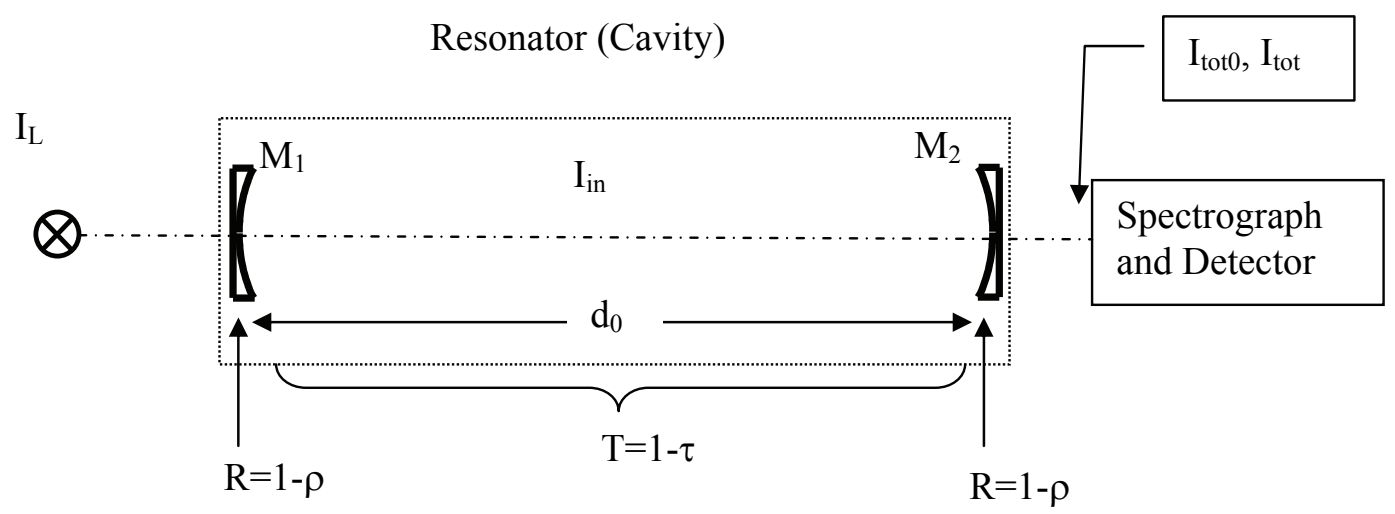

Fig. 2. Sketch of intensities vs. wavelength: $I_{\text {in } 0}\left(n_{0}\right)$ : intensity (after $n_{0}$ passes through the cavity) in the pure air filled cavity without any absorbers, $I_{\text {in } 0}^{\prime}(n)$ : intensity (after $n$ passes through the cavity) after any continuous absorption (due to gases or aerosol) has taken place. $I_{\text {in }}$ $(n)$ : Intensity after $n$ passes through the cavity including also differential absorptions (note that $n$ varies with wavelength since the trace gas absorption cross section varies with wavelength)). $I_{\text {in }}\left(n_{0}\right)$ : Theoretical intensity for the same absorptions if the number of traverses were not reduced.

With:

$\sigma=$ Absorption cross section of the trace gas

$\bar{c}=$ Average trace gas concentration in the cavity

$d_{0}=$ Length of the cavity (resonator)

The average number of passes through the cavity $\bar{n}$, which the photons travel and the average light path $\bar{L}$, results in the decay of the initial intensity inside the cavity $I_{i n}(0)$ to the remaining intensity $I_{\text {in }}(\bar{n})=1 / e \cdot I_{\text {in }}(0)$.

Thus, we have from Eq. (15):

$\ln \left(\frac{I_{\text {in }}(0)}{I_{\text {in }}(\bar{n})}\right)=\ln e=1 \approx \bar{n} \cdot\left(\tau+\rho_{0}\right)$

With the average number of traverses of the photons through the cavity and associated average length of the light path we finally derive for the length of the average light path $\bar{L}$ :

$\bar{n} \approx \frac{1}{\tau+\rho_{0}}$ and $\bar{L}=d_{0} \cdot \bar{n} \approx \frac{d_{0}}{\tau+\rho_{0}}=\frac{d_{0}}{\sigma \cdot \bar{c} \cdot d_{0}+\rho_{0}}$

Note that $\tau$ includes all extinction processes in the cavity i.e. aerosol absorption and Mie scattering as well as absorption by trace gases, except those by pure air due to absorption and Rayleigh scattering (which are taken into account by using $\rho_{0}$ instead of $\rho$, see above).

For an empty (i.e. air filled) cavity we therefore have:

$\bar{L}_{0}=\frac{d_{0}}{\rho_{0}}$

Where the index 0 always corresponds to the cavity without absorbers.

If additional (broadband) extinction is assumed, the average length of the absorption path $\bar{L}$ will be approximately:

$\bar{L}=\frac{d_{0}}{\rho_{0}+\varepsilon_{B} d_{0}}$

where $\varepsilon_{B}$ denotes all broadband extinctions except Rayleigh scattering, (e.g. due to Mie scattering and broadband trace gas absorption) and $d_{0}$ is the length of the resonator (see Fig. 2).

In practice the length of the average light path $\left(\bar{L}_{0}\right)$ can be determined by different techniques e.g. by measuring the ring-down time of the cavity only filled with filtered air (Meinen et al., 2008), or by addition of a different gas with known Rayleigh scattering cross section (Washenfelder et al., 2008).

The following examples illustrate the consequences of the above considerations:

1) In a cavity $\left(d_{0}=0.5 \mathrm{~m}, \quad R_{0}=0.99995\right.$ and thus $\left.\rho=5 \times 10^{-5}\right)$ without trace gas $(\tau=0)$ according to Eq. (18) one would obtain an average light path of $\bar{L}_{0}(\tau=0)=10^{4} \mathrm{~m}$ or $10 \mathrm{~km}$ (neglecting the contribution from Rayleigh scattering, which would amount to $\varepsilon_{R} d_{0} \approx 2.55 \times 10^{-6}$ at $622 \mathrm{~nm}$ (Penndorf, 1957), i.e. for simplicity taking $\rho=\rho_{0}$ here).

2) Now adding an absorber with $\tau=5 \times 10^{-5}$ to the same cavity we obtain an average light path of $\bar{L}\left(\tau=5 \times 10^{-5}\right)=$ $5 \times 10^{3} \mathrm{~m}$ or $5 \mathrm{~km}$.

Note that in a high finesse resonator (i.e. one with high mirror reflectivity) a rather weak absorber with $\tau=d_{0} \cdot \varepsilon=$ $5 \times 10^{-5}$ or $\varepsilon=10^{-4} \mathrm{~m}^{-1}$ is already sufficient to significantly reduce the effective average light path. CRDS measurements are based on this light path reduction. Moreover this implies for broad band cavities, if $\tau=\tau\left(\lambda_{\max }\right)$ in the above example denotes the absorption in the centre of an absorption line, then the average light path length may be quite different at any other point of the spectrum (this effect is discussed in more detail in Sect. 4).

Considering an absorption band as sketched in Fig. 1 we have after $\mathrm{n}$ traverses of the cavity (for the moment neglecting any broadband absorption, i.e. $\left.I_{\mathrm{in} 0}(n)=I_{\mathrm{in} 0}^{\prime}(n)\right)$ :

$I_{\mathrm{in}}(n)=I_{\mathrm{in} 0}(n) \cdot e^{-\sigma \cdot \bar{c} \cdot d_{0} \cdot n}$ 
Where $I_{\text {in } 0}(n)$ denotes the intensity in the absence of the absorption band. According to DOAS principles (Platt, 1994; Platt and Stutz, 2008) $I_{\text {in } 0}(n)$ (in the figure this is $I^{\prime} \operatorname{in}_{0}(n)$ ) can be thought to be interpolated from the intensity outside the absorption band as illustrated in Fig. 1. Only if the number of round-trips $n$ is constant with - and without absorber the optical density according to the trace gas to be measured (from Lambert-Beer absorption) can be written as:

$D=\ln \left[\frac{I_{\text {in } 0}(n)}{I_{\text {in }}(n)}\right]=\sigma \cdot \bar{c} \cdot d_{0} \cdot n$

From Eq. (22) the trace gas concentration inside the cavity $\bar{c}$ can be calculated once the absorption cross section $\sigma$ is known (usually from laboratory measurements), the number of round trips is constant, and $L$ (or $n$ ) is known. Outside the cavity, i.e. at the detector all intensities are lower by a factor $1-R_{0}=\rho_{0}$ :

$\rho_{0} \cdot I_{i n}(n)=\rho_{0} \cdot I_{i n 0}(n) \cdot e^{-\sigma \cdot \bar{c} \cdot L}$

leading to the same equation for $D$ as given in Eq. (22).

Further consequence of effect of a reduced light path due to absorbers in the cavity is a different measured optical density $D_{C E}$ with a cavity what is illustrated in the following. The optical density seen by the detector through the second mirror of the cavity, $D_{C E}$, after n traverses is given by:

$D_{C E}=\ln \left(\frac{I_{\mathrm{tot} 0}}{I_{\mathrm{tot}}}\right)=\ln \left(\frac{\int_{0}^{\infty} I_{\mathrm{in} 0}(n) d n}{\int_{0}^{\infty} I_{\mathrm{in}}(n) d n}\right)$

$I_{\text {tot }}, I_{\text {tot0 }}$ denote the intensities at the detector with and without an absorber, respectively. Here, again we treat $n$ as a continuous variable, which in reality can only assume discrete values. (A derivation with discrete $n$ values, is also possible and leads to the same result, see e.g. Fiedler, 2003). Note that we can treat the decay of intensity independent from continuous input of radiation into the cavity by following a pulse of radiation with infinitesimal duration, which will behave according to the above equation. The only prerequisite is linear superposition of intensities, which is given in the case of incoherent radiation considered here (but not necessarily when treating coherent input to the cavity). Therefore the continuous input can be described as a train of very short pulses into the cavity.

Using Lambert-Beer's law we can further rewrite the above equation:

$$
\begin{aligned}
D_{C E} & =\ln \left(\frac{\int_{0}^{\infty} I_{\mathrm{in} 0}(n) d n}{\int_{0}^{\infty} I_{\mathrm{in}}(n) d n}\right) \\
& =\ln \left(\frac{\int_{0}^{\infty} I_{\mathrm{in}}(0) \cdot e^{-\rho_{0} n} d n}{\int_{0}^{\infty} I_{\mathrm{in}}(0) \cdot e^{-\left(\rho_{0}+\tau\right) n} d n}\right)
\end{aligned}
$$

The initial intensity $I_{\text {in }}(0)$ is a (non-zero) constant and can be taken out of the integral in the numerator and denominator.
Then evaluating the integral yields:

$$
\begin{aligned}
D_{C E} & =\ln \left(\frac{\int_{0}^{\infty} e^{-\rho_{0} n} d n}{\int_{0}^{\infty} e^{-\left(\rho_{0}+\tau\right) n} d n}\right) \\
& =\ln \left(\frac{-\left.\frac{1}{\rho_{0}} e^{-\rho_{0} n}\right|_{0} ^{\infty}}{-\left.\frac{1}{\rho_{0}+\tau} e^{-\left(\rho_{0}+\tau\right) n}\right|_{0} ^{\infty}}\right) \\
& =\ln \left(\frac{\rho_{0}+\tau}{\rho_{0}}\right)=\ln \left(1+\frac{\tau}{\rho_{0}}\right)
\end{aligned}
$$

This is the trace gas optical density (or simply the DOAS signal) from the CE-DOAS measurement. We illustrate the consequences of Eq. (26) for two extreme (but realistic) cases:

1) For small values of $\rho_{0} \ll \tau$ (or effective mirror reflectivities $R=1-\rho_{0}$ close to unity, i.e. very good mirrors) we obtain for the optical density due to extinction in the cavity (Eq. 26):

$D_{C E}=\ln \left(\frac{\tau+\rho_{0}}{\rho_{0}}\right) \underbrace{\approx}_{\rho_{0} \ll \tau} \ln \left(\frac{\tau}{\rho_{0}}\right) \underbrace{\propto}_{\rho_{0}=\text { const. }} \ln (\tau)=\ln \left(\alpha d_{0}\right)(27)$

Thus the above equation yields $D_{C E} \propto \ln \tau$ being nearly constant. In other words, the trace gas optical density $D_{C E}$ will only be weakly dependent on the extinction (e.g. due to trace gases) and thus the trace gas concentration $\bar{c}$ in the cavity.

2) For large values of $\rho_{0} \gg \tau$ (i.e. relatively poor mirror reflectivities and/or high Rayleigh extinctions $R_{0}=1-\rho_{0}$ or small trace gas absorption) we have:

$D_{C E}=\ln (\underbrace{\frac{\rho_{0}+\tau}{\rho_{0}}}_{\approx 1}) \approx \frac{\rho_{0}+\tau}{\rho_{0}}-1=\frac{\tau}{\rho_{0}} \propto \tau$

Since $\rho_{0}$ is constant for a given set-up $D_{C E}$ will be proportional to $\tau=\sigma \cdot \bar{c} \cdot d_{0}$. Only in the latter case (i.e. poor mirror reflectivities or small trace gas absorption) the measured optical density $D_{C E}$ will indeed be proportional to the trace gas absorption $\tau$ and thus the trace gas concentration $\bar{c}$.

From Eq. (28) we calculate the effective (i.e. intensity weighted average) CE-DOAS light path $\bar{L}_{\text {eff }}=\bar{L}_{\text {eff }}(\tau, \rho)$, which corresponds to an absorption light path of a Lambert Beer absorption (e.g. LP-DOAS) with the same optical density:

$\alpha_{C E}=\frac{D_{C E}}{\bar{L}_{\mathrm{eff}}}=\alpha=\frac{\ln \left(\frac{\rho_{0}+\tau}{\rho_{0}}\right)}{\bar{L}_{\mathrm{eff}}}=\frac{\tau}{d_{0}}=\sigma \cdot c$

$\bar{L}_{\mathrm{eff}}=\frac{\ln \left(\frac{\rho_{0}+\tau}{\rho_{0}}\right)}{\tau} d_{0}$

The effective light path corresponds to an effective number of reflections $n_{\text {eff }}$ via:

$\bar{L}_{\text {eff }}=n_{\text {eff }} \cdot d_{0}$ or $n_{\text {eff }}=\frac{\ln \left(\frac{\rho_{0}+\tau}{\rho_{0}}\right)}{\tau}$ 


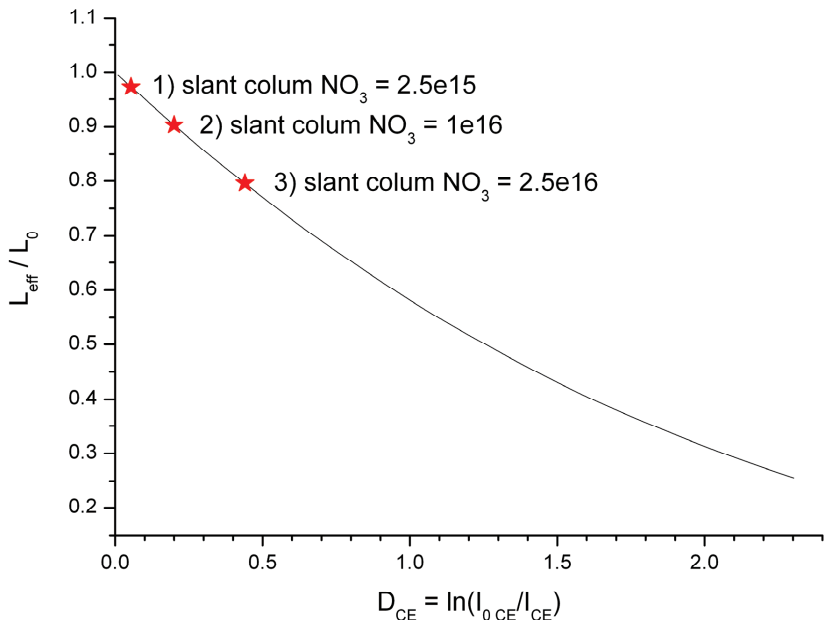

Fig. 3. Sketch of CEAS set-up. The optical resonator is formed by two concave mirrors $M_{1}$ and $M_{2}$, both with the same reflectivity $R=1-\rho$. The transmission factor for one traverse through the cavity is $T=1-\tau=1-\varepsilon d_{0}$. The intensity of the radiation emitted by the source is $I_{L}$ (transfer optics between light source and resonator is not shown).

For small absorptions, i.e. empty cavity $(\tau \rightarrow 0$ i.e. $T \rightarrow 1$ ) we derive the average light path for an empty cavity, $L_{0}$ :

$\bar{L}_{\text {eff }}(\tau \rightarrow 0)=\bar{L}_{0}=\frac{1}{\rho_{0}} d_{0} \quad$ or $\rho_{0}=\frac{d_{0}}{\bar{L}_{0}}$

With the above relationship we can write the effective light path length $\bar{L}_{\text {eff }}$ as function of the light path length of the empty (air filled) cavity

$\bar{L}_{\text {eff }}=\frac{\ln \left(1+\frac{\tau}{\rho_{0}}\right)}{\tau} d_{0}=\frac{\ln \left(1+\frac{\tau \cdot \bar{L}_{0}}{d}\right)}{\tau} d_{0}$

Introducing $D_{C E}$ from above (Eq. 26)

$D_{C E}=\ln \left(1+\frac{\tau}{\rho_{0}}\right)$ or $e^{D_{C E}}-1=\frac{\tau}{\rho_{0}}$ or

$\left(e^{D_{C E}}-1\right) \rho_{0}=\tau$

we obtain:

$\bar{L}_{\text {eff }}=\frac{D_{C E}}{\left(e^{D_{C E}}-1\right) \rho_{0}} \cdot d_{0}=\frac{D_{C E}}{\left(e^{D_{C E}}-1\right)} \bar{L}_{0}$

The dependence of $\bar{L}_{\text {eff }}$ on $D_{C E}$ is illustrated in Fig. 3. For illustration the effective light paths at $662 \mathrm{~nm}$ (peak of the $\mathrm{NO}_{3}$ absorption band) for three realistic $\mathrm{NO}_{3}$ total column densities in the cavity are also shown (see Meinen et al., 2008). Note that the ratio $L_{\text {eff }} / L_{0}$ only depends on the trace gas column density in the cavity (which, of course, is a function of $c, d_{0}$, and $R$ ). At this point we define the effective optical density $D_{\text {eff }}$ as:

$D_{\text {eff }}=\alpha \cdot \bar{L}_{0}$ with (see Eq. 29):

$\alpha=\frac{D_{C E}}{\bar{L}_{\text {eff }}}$ and $\bar{L}_{\text {eff }}=\frac{D_{C E}}{\left(e^{D_{C E}}-1\right)} \bar{L}_{0}$

we obtain by introducing $\bar{L}_{\text {eff }}$ from Eq. (34);

$$
\begin{aligned}
D_{\text {eff }} & =\alpha \bar{L}_{0}=\frac{D_{C E}}{\bar{L}_{\text {eff }}} \cdot \bar{L}_{0}=D_{C E} \cdot \frac{\left(e^{D_{C E}}-1\right)}{D_{C E} \cdot \bar{L}_{0}} \cdot \bar{L}_{0} \\
& =e^{D_{C E}}-1=\frac{I_{0 \mathrm{tot}}}{I_{\text {tot }}}-1
\end{aligned}
$$

We obtain for the trace gas absorption coefficient:

$\alpha(\lambda)=\left(\frac{I_{0 \mathrm{ges}}}{I_{\mathrm{ges}}}-1\right) \cdot \underbrace{\frac{1-R(\lambda)}{d}}_{1 / \bar{L}_{0}}=\sigma(\lambda) \cdot \bar{c}+\varepsilon_{\mathrm{Mie}}$

Note that this relationship between $D$ and intensities of empty/filled cavity was also derived by Fiedler et al. (2003). Thus, BB-CEAS contains the presented correction, but has to rely on stable $I_{0}$ conditions.

Further rewriting $D_{\text {eff }}$ we can derive an alternative form of the effective light path:

$D_{\text {eff }}=\bar{c} \cdot \sigma \cdot \overline{L_{0}}=\left(\frac{I_{\text {in } 0}}{I_{\text {in }}}-1\right)=\exp \left(\bar{c} \cdot \sigma \cdot L_{\text {eff }}\right)-1$

This is the equation which we use for further analysis.

Solving the above Eq. (38) for $L_{\text {eff }}$ yields:

$\bar{L}_{\mathrm{eff}}=\frac{\ln \left(\bar{c} \cdot \sigma \cdot \overline{L_{0}}+1\right)}{\bar{c} \cdot \sigma}$

Note that for small optical densities, i.e. $\bar{c} \cdot \sigma \cdot \overline{L_{0}} \ll 1, L_{\mathrm{eff}}$ approaches $L_{0}$ :

$\bar{L}_{\text {eff }} \underbrace{\approx}_{\bar{c} \cdot \sigma \cdot \overline{L_{0}} \ll 1} \frac{\bar{c} \cdot \sigma \cdot \overline{L_{0}}-1+1}{\bar{c} \cdot \sigma}=\overline{L_{0}}$

A consequence of this finding is that a too high mirror reflectivity (compared to the extinction losses in the cavity) can cause problems. (This is also true for BB-CEAS, the equation given by Fiedler et al. (2003) just already contains these corrections and thus does not explicitly show the non-linear dependency between reflectivity and concentration, the nonlinearity is expressed as a reduction of the Q-Factor for high concentrations.) On the other hand, however, a high mirror reflectivity is a prerequisite for long light paths and thus high sensitivity. Therefore, the reflectivity $R_{0}$ cannot usually be made so small (or $\rho_{0}$ so large) that approximation 2 (see equation 28 above) becomes valid. An optimum strategy therefore, would involve a combination of high reflectivity cavity and correction of the observed optical densities for the effect of light path reduction. In the following, we investigate the implications of this strategy. 


\section{Correction for light path reduction due to extinction in the cavity}

In the following we present solutions for the problem of determining the effective average length of the light path $\mathrm{L}_{e f f}$ in the presence of absorbers in the cavity.

In principle, there are several possibilities:

1. Determine the average light path $\bar{L}_{0}$ with an empty, i.e. air filled, cavity (where replacing $R$ by $R_{0}$ accounts for the known Rayleigh extinction of air as explained above) and use the corrected effective light path length $\bar{L}_{\text {eff }}$ (see Eq. 34) instead of $L_{0}$ for the calculation of the average trace gas concentration $\bar{c}$ from the measured $D_{C E}$.

2. Alternatively one may use the "empty cavity" $L_{0}$ to calculate a concentration $c_{0}$ from $D_{C E}$ and then apply a correction factor on the concentration (taking the ratio of $L_{0} / L_{\text {eff }}$ from Eq. 34).

$$
\bar{c}_{0}=\frac{D_{C E}}{\sigma \cdot \bar{L}_{0}}, \bar{c}=\frac{\bar{L}_{0}}{\bar{L}_{\mathrm{eff}}} c_{0}=\frac{e^{D_{C E}}-1}{D_{C E}} \cdot c_{0}
$$

3. Another possibility is to convert the trace gas absorption cross section $\sigma$ to a corrected value $\sigma_{\text {eff }}$, which is then used in the DOAS fit and in conjunction with $L_{0}$ to calculate the concentration $\bar{c}$ from $D_{C E}$ (again taking the ratio of $L_{0} / L_{\text {eff }}$ from Eq. 34):

$$
\begin{aligned}
D_{C E}(\lambda) & =\sigma(\lambda) \cdot \bar{c} \cdot \bar{L}_{\mathrm{eff}}(\lambda)=\sigma_{\mathrm{eff}}(\lambda) \cdot \bar{c} \cdot \bar{L}_{0} \\
\sigma_{\mathrm{eff}}(\lambda) & =\sigma(\lambda) \cdot \frac{\bar{L}_{\mathrm{eff}}(\lambda)}{\bar{L}_{0}}=\sigma(\lambda) \cdot \frac{D_{C E}}{e^{D_{C E}-1}}
\end{aligned}
$$

4. Finally, the measured optical density $D_{C E}$ may be corrected to obtain a corrected optical density $D_{\text {eff }}$ according to Eq. (36):

$D_{\text {eff }}=e^{D_{C E}}-1=\frac{I_{0 \mathrm{ges}}}{I_{\mathrm{ges}}}-1$

This procedure corresponds to a linearisation of the problem, since $D_{\text {eff }}(\lambda)$ is proportional to the absorption cross section $\sigma(\lambda)$, thus a "normal" DOAS fit of $D_{\text {eff }}(\lambda)$ to $\sigma(\lambda)$ can be performed as in classic DOAS approaches with constant light path length.

All four correction factors will depend on the mirror reflectivity $R_{0}$ and the extinction in the cavity including the extinction due to the trace gas optical density. Since the true trace gas optical density is a priori unknown (as the true $I_{0}$ is unknown), a theoretical value for $I_{0}$ has to be assumed (e.g. if light source and adjustment is constant the $I_{0}$ measurement from a pure air-filled cavity can be used) or an iterative procedure has to be applied like the one mentioned at the end of Sect. 5.
Approaches a and b, which use the measured $D_{C E}$ have the problem that the shape of the trace gas absorption bands will be distorted due to the reduction of the light path by the trace gas absorption as discussed in Sect. 5 below and illustrated in Fig. 4. Only at small optical densities $D_{C E} \ll 1$ this effect will become negligible.

\section{Distortion of absorption bands due to wavelength- dependent extinction}

From Eq. (26) it follows that the (differential) optical density varies as:

$D_{C E}=\ln \left(\frac{\rho_{0}+\tau}{\rho_{0}}\right)$

Thus $D_{C E}(\lambda)$ is not a linear function of the (differential) absorption cross section $\sigma(\lambda)$, while in conventional DOAS (or any conventional absorption spectroscopy) we have direct proportionality between the optical density $D(\lambda)$ and $\sigma(\lambda)$ as shown in Eq. (22).

In the case of $\rho_{0} \ll \tau$ and thus $\rho_{0} / \tau \ll 1$ (and neglecting the wavelength dependence of $\rho_{0}$ ) Eq. (44) can be linearised to approximately yield proportionality between $D(\lambda)$ and $\sigma(\lambda)$ as shown above (Eq. 28). However, as discussed above this condition is frequently not fulfilled. Therefore, if not $\rho \ll \tau$, there will be a distortion of $D_{C E}(\lambda)$ in the sense that $D_{C E}(\lambda)$ is somewhat smaller at larger optical densities than given by the linear relationship (Eq. 22) i.e. in the centre of an absorption band leading to a change in the shape of the measured absorption spectrum. An example of this behaviour is shown in Fig. 4.

Using the linear relationship of Eq. (22) to analyse the detected absorption will cause errors in the retrieved optical density of trace gases as illustrated in Fig. 4. Thus not only the derived concentration will be incorrect, but also the measurement error increases. This can become a particular problem if the features of several absorbers overlap. Instead of correcting the light path for the centre of the absorption line, we can modify the absorption cross section, which will be used for the analysis (see Sect. 4, point 3).

Instead of using the original absorption cross section $\sigma(\lambda)$ with the corrected effective light path, the original determined average light path is used with a modified (corrected) absorption cross section $\sigma_{\text {eff }}(\lambda)$. So the modified absorption cross section is changing in the same way with wavelength as the light path length does. In the following, we give an example based on recent measurements of nitrate radicals by CE-DOAS (Meinen et al., 2008). We consider the changing absorption structure of $\mathrm{NO}_{3}$ around $662 \mathrm{~nm}$ at two different $\mathrm{NO}_{3}$ column densities:

1. High $\mathrm{NO}_{3}$ Column: $1 \times 10^{16} \mathrm{~cm}^{-2}$ (corresponding to a concentration: $c=1 \times 10^{10} \mathrm{~cm}^{-3}$ or about $400 \mathrm{ppt}$ at room temperature and standard pressure at $\bar{L}_{0}=10^{6} \mathrm{~cm}$ $(10 \mathrm{~km}))$. 

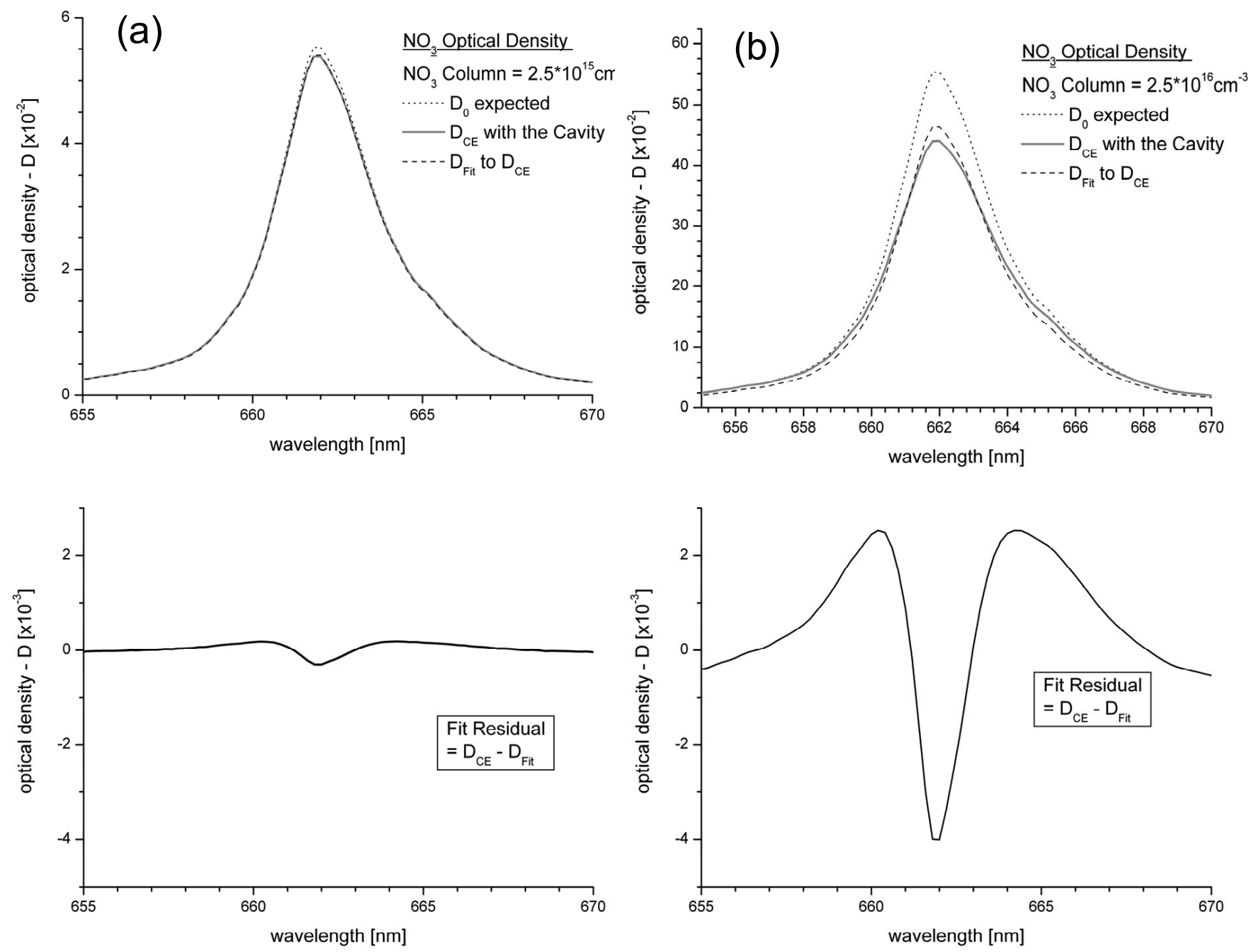

Fig. 4. The effect of the distortion of absorption bands due to different light paths in the band centre and at the wings. $\mathrm{Here}$ an $\mathrm{NO}_{3}$ band at $662 \mathrm{~nm}$ is used as an example. (a) At low optical densities $D \ll 1\left(\mathrm{NO}_{3}\right.$ column $\left.2.5 \times 10^{15} \mathrm{~cm}^{-2}\right)$ the effect of changing absorption structure is rather small, the actual line shape $D_{C E}(\lambda)$ fits the expected line shape calculated for $D$ being independent of wavelength quite well. The arising residual (lower panel) is small and will not significant increase the measurement error. (b) At higher optical densities $\left(\mathrm{NO}_{3}\right.$ column $10 \times 10^{15} \mathrm{~cm}^{-2}$ ) the distortion in the shape of the absorption structure becomes clearly visible and leads to errors in the fit of the $\mathrm{NO}_{3}$ absorption cross section (upper panel). The expected optical density $D_{0}$ is significantly different from the detected optical density $D_{C E}(\lambda)$ actually retrieved. The rather large fitting residual (lower panel) indicates an enhanced measurement error.

2. Moderate $\mathrm{NO}_{3}$ Column: $2.5 \times 10^{15} \mathrm{~cm}^{-2}$ (corresponding to $c=2.5 \times 10^{9} \mathrm{~cm}^{-3}(100 \mathrm{ppt})$ at the same conditions as above).

The results are shown in Fig. 4. It can be seen that at high optical densities the distorted shape of the absorption structure, leads to considerable errors in the DOAS-fit of the $\mathrm{NO}_{3}$ absorption cross section (Fig. 4b, upper panel).

An even more severe consequence of distorted bands will be apparent if absorbers with overlapping spectra are present, which is quite normal for DOAS application. Since a strong absorber will show distorted spectra it can (without proper correction measures) not be fitted properly, thus large residuals remain, which severely limit the ability of the system to determine weaker absorbers. While in classical (constant pathlength) DOAS applications the ability to detect weak absorbers is usually limited by the noise in the spectrum in CEDOAS the detection limit for weak absorbers can be much degraded.

A realistic case would be detection of HONO (nitrous acid) in the near UV (approx. 350-400 nm), which constitutes the absorber with weak structures. Here the strong absorber is $\mathrm{NO}_{2}$, typically $\mathrm{HONO} / \mathrm{NO}_{2}$ is a few percent (see e.g. Alicke et al., 2002). Since the HONO differential absorption cross section in the above wavelength range is about three times larger than that of $\mathrm{NO}_{2}$ the $D^{\prime}$ due to $\mathrm{HONO}$ is usually less than $10 \%$ of $D^{\prime}$ due to $\mathrm{NO}_{2}$. Thus a $10 \%$ 
distortion of the $\mathrm{NO}_{2}$ band as a result to a $\mathrm{NO}_{2}$ optical density of about 0.2 would lead to a residual of the order of the HONO signal thus rendering HONO detection impossible.

The phenomenon of band distortion is - in principle - not unknown in DOAS applications where non-linear variation of the optical density with wavelength can occur for different reasons (Platt et al., 1997; Platt and Stutz, 2008). Several techniques were developed to compensate for these effects. For instance, there is the effect of insufficient spectral resolution (e.g. Volkamer et al., 1998; Maurellis et al., 2000) or the effect of wavelength dependent airmass factors (Marquard et al., 2000) in the case of strong absorbers. In the former case, high-resolution modelling of Lambert-Beer's law (Volkamer, 2001) or IMAP-DOAS (Frankenberg et al., 2005) are solutions, although further approaches were suggested. In the latter case "modified DOAS" (Coldewey-Egbers et al., 2004) proved successful to solve the problem. These widely used techniques can also be applied to compensate for the non-linearity encountered in CE-DOAS although the origin of the non-linearity is different from the known cases.

\section{Practical Determination of the correction (e.g. length of the effective light path)}

The above derived correction formulae (Eqs. 34, 41, 42, 43) for deriving a effective light path or to correct the derived concentration etc. are straightforward to apply if the total optical density $D_{C E}$ due to the total extinction (except extinction due to Rayleigh scattering) in the cavity is known. Unfortunately, it can be difficult to determine this quantity, which is composed of:

1. Total extinction due to all trace gases present in the cavity.

\section{Extinction due to Mie scattering}

\section{Extinction due to turbulence in the cavity}

As explained above the term $D_{C E}$ in the correction formulae (Eqs. 34, 41, 42), respectively, requires the total optical density $D_{C E}$, while the DOAS analysis of the spectra only provides the differential optical density $D_{C E}^{\prime}$. Therefore, the application of the above correction is not straightforward in general, if $I_{0}$ is not known. Similar problems arise if data analysis is performed with the BB-CEAS equations presented by Fiedler et al. (2005). However, there have been a number of techniques developed to solve this problem:

1. If there are only known absorbers, their total absorption $\sigma \cdot c$ can be calculated from their (measured) differential absorption $\sigma^{\prime} \cdot c$ since the ratio of $\sigma(\lambda) / \sigma^{\prime}(\lambda)$ is known. There remains, however the problem of possible Mie extinction.

2. The total extinction in the cavity can be determined from the ring-down time (see Meinen et al., 2008).
The problem associated with this approach is the possible variation of the mirror reflectivity $R(\lambda)$ with wavelength. While this causes no problem for (nearly) monochromatic laser sources frequently used for CRDS measurement, the broadband operation (e.g. using LED sources) in CE-DOAS will lead to the different wavelengths having different ring-down times. Thus, the signal seen by the (broadband) detector consists of a continuum of exponential decaying functions with different decay times, which do not follow a single exponential decay. This problem can be overcome by modelling the intensity in the cavity as a function of time as described by Meinen (2007) and Meinen et al. (2008), however at the expense of an increased uncertainty in $R_{0}$.

3. The total extinction in the cavity can also be determined from the reduction in intensity when changing from a pure air filled cavity to an ambient air filled one (e.g. Thieser, 2008). The drawbacks here are the possible change of the cavity quality between measurement and the additional hardware required for periodically sealing the cavity from the ambient air and flushing it with pure air.

In fact, there are at least three potential causes for changes in the total intensity received at the detector:

(a) De-adjusting of cavity/optics

(b) Degradation of mirrors (by dust etc.)

(c) Light source instability

The problem is that in a practical situation the actual cause for intensity reduction will usually be unknown. However the consequences of an intensity reduction will be quite different: In cases a) and b) the light path length will actually be reduced, while in case a) it will not (in fact, a reduction of light source intensity will not affect CE-DOAS evaluations as long as the signal to noise ratio is not too much degraded).

4. The effective path length can be determined by determining the differential strength of absorption band(s) $D_{C E, K}$ due to species $X_{K}$ with known column density per unit length (e.g. of $\mathrm{H}_{2} \mathrm{O}, \mathrm{O}_{2}$, or $\mathrm{O}_{4}$ ). Since both, $D_{\text {eff }}$ and $D_{C E, K}$ are known (the former is measured, the latter is calculated from concentration and absorption cross section (or the product of both quantities in the case of $\mathrm{O}_{4}$ ).) the optical density $D_{B}$ due to broadband absorption (i.e. aerosol optical density) can be determined. This technique has been employed by many DOAS applications (e.g. Platt and Stutz, 2008) and was introduced to CEAS by Ball et al. (2004). Also for the analysis of the concentration of the known absorbers the distortion of absorption band shapes has to be considered as described in Sect. 4. 
5. Another approach is the selective removal of the trace gas $\mathrm{X}$ to be measured (in the case of $\mathrm{NO}_{3}$ by e.g. NOaddition or photolysis (see e.g. Brown et al., 2002), then $\bar{L}$ in the presence of all continuous absorbers except species $\mathrm{X}$ can be determined.

6. The effect of Mie scattering can also be neglected in cases where the air is aerosol-filtered during the measurements (e.g. Brown et al., 2001, 2002). However, frequently this is not desirable, since aerosol filters may - depending of its chemical nature - affect concentration of the trace gas to be measured.

\section{The optimum mirror reflectivity for CE-DOAS}

We showed above that the highest mirror reflectivity $R_{0}$ is not the best choice for two reasons:

1. The DOAS signal e.g. the measured differential optical density $D_{C E}^{\prime}$ due to the trace gas will not grow in proportion to $1 / \rho_{0}=1 /\left(1-R_{0}\right)$ as one would expect from the variation of the average light path of the (empty) cavity being proportional to $1 / \rho$. In fact $D_{C E}^{\prime}$ grows less than $1 / \rho_{0}$ with improved mirror reflectivity unless $\rho_{0}$ is much larger than $\tau=\sigma \cdot \bar{c} \cdot d_{0}$.

2. Another factor to keep in mind is the signal to noise ratio of the measured spectrum. Since the signal reaching the detector $I=\rho \cdot I_{\text {in }}$ (see Eq. 23) is proportional to $\rho=1-R$ it diminishes as $R$ approaches unity. Assuming the detector noise to be dominated by photon shot noise which is proportional to $I^{1 / 2}$ the signal to noise ratio will vary as $I^{-1 / 2}$ and one can derive an optimal $\rho_{\mathrm{optS}}=2 \cdot \varepsilon_{\mathrm{tot}}$ (e.g. Fiedler, 2005). Where $\varepsilon_{\text {tot }}$ denotes the absorption due to the trace gas.

Combining both arguments one arrives at an optimum mirror reflectivity below $\rho_{\text {opts}}$. However, in practice the mirror reflectivity has to be chosen beforehand, when the actual trace gas concentrations are still unknown, so the mirror reflectivity can only be approximately optimal and the method proposed by Fiedler et al. (2007) is a sufficient approximation.

\section{Summary}

We investigate the relationships between mirror reflectivity and BB-CEAS signal in detail and conclude that it is usually not appropriate to operate instruments in a range where $\rho_{0}$ is much larger than $\tau=\sigma \cdot \bar{c} \cdot d_{0}$ and thus the differential optical $D_{C E}^{\prime}$ observed with the cavity enhanced instrument is in good approximation proportional to the trace gas concentration $\bar{c}$. Therefore, in reality corrections have to be performed to compensate for the reduced (and variable) light path in the presence of variable (broadband and narrow band) atmospheric extinction. The determination of the extinction is not always straightforward; however, we review a number of techniques to determine atmospheric extinction and correct for this effects.

Since a large fraction of the atmospheric extinction can be due to aerosol, these techniques (e.g. determination of the differential optical density due to trace gases with known absorption like $\mathrm{O}_{2}$ or $\mathrm{O}_{4}$ ) also provide means to derive the absolute aerosol extinction from only relative DOAS measurements.

In summary, we present methods to combine the advantages of two highly successful techniques: The compact design of high-finesse cavity based absorptions spectroscopy with the robustness and specificity of the DOAS technique.

Edited by: A. Zahn

\section{References}

Alicke, B., Platt, U., and Stutz, J.: Impact of nitrous acid photolysis on the total hydroxyl radical budget during the Limitation of Oxidant Production/Pianura Padana Produzione di Ozono study in Milan, J. Geophys. Res., 107(D22), 8196, doi:10.1029/2000JD000075, 2002.

Ball, S. M., Langridge, J. M., and Jones, R. L.: Broadband cavity enhanced absorption spectroscopy using light emitting diodes, Chem. Phys. Lett., 398, 68-74, 2004.

Bitter, M., Ball, S. M., Povey, I. M., and Jones, R. L.: A broadband cavity ringdown spectrometer for in-situ measurements of atmospheric trace gases, Atmos. Chem. Phys., 5, 2547-2560, 2005, http://www.atmos-chem-phys.net/5/2547/2005/.

Brown, S. S., Stark, H., Ciciora, S. J., and Ravishankara, A. R.: In-situ measurement of atmospheric $\mathrm{NO}_{3}$ and $\mathrm{N}_{2} \mathrm{O}_{5}$ via cavity ring-down spectroscopy, Geophys. Res. Lett., 28(17), 32273230, 2001.

Brown, S. S., Stark, H., Ciciora, S. J., McLaughlin, R. J., and Ravishankaraa, A. R.: Simultaneous in-situ detection of atmospheric $\mathrm{NO}_{3}$ and $\mathrm{N}_{2} \mathrm{O}_{5}$ via cavity ring-down spectroscopy, Rev. Sci. Instrum., 73(9), 3291-3301, 2002.

Brown, S. S.: Absorption spectroscopy in high-finesse cavities for atmospheric studies, Chem. Rev., 103(12), 5219-5238, 2003.

Coldewey-Egbers, M., Weber, M., Buchwitz, M., and Burrows, J. P.: Application of a modified DOAS method for total ozone retrieval from GOME data at high polar latitudes, Adv. Space Res., 34(4), 749-753, 2004.

Engeln, R., Berden, G., Peeters, R., and Meijer, G.: Cavity enhanced absorption and cavity enhanced magnetic rotation spectroscopy, Rev. Sci. Instrum., 69, 3763-3769, 1998.

Englund, D. R.: Cavity-Enhanced Absorption Spectroscopy of BChla, B. Sc. Thesis, California Institute of Technology, Pasadena, California, 2002.

Fawcett, B. L., Parkes, A. M., Shallcross, D. E., and Orr-Ewing, A. J.: Trace detection of methane using continuous wave cavity ring-down spectroscopy at $1.65 \mu \mathrm{m}$, Phys. Chem. Chem. Phys., 4, 5960-5965, 2002.

Fiedler, S. E., Hese, A., and Ruth, A. A.: Incoherent broad-band cavity-enhanced absorption spectroscopy, Chem. Phys. Lett., 371, 284-294, 2003. 
Fiedler, S. E.: Incoherent Broad-Band Cavity-Enhanced Absorption Spectroscopy, PhD Thesis, D83 Faculty II - Mathematics and Sciences, Technische Universität Berlin, 2005.

Fiedler, S. E., Hese, A., and Heitmann, U.: Influence of the cavity parameters on the output intensity in incoherent broadband cavity-enhanced absorption spectroscopy, Rev. Sci. Instr., 78, 073104, doi:10.1063/1.2752608, 2007.

Gherman, T., Venables, D. S., Vaughan, S., Orphal, J., and Ruth, A. A.: Incoherent Broadband Cavity-Enhanced Absorption Spectroscopy in the near-Ultraviolet: Application to $\mathrm{HONO}$ and $\mathrm{NO}_{2}$, Environ. Sci. Technol., 42, 890-895, 2008.

Frankenberg, C., Platt, U., and Wagner, T.: Iterative maximum a posteriori (IMAP)-DOAS for retrieval of strongly absorbing trace gases: Model studies for $\mathrm{CH}_{4}$ and $\mathrm{CO}_{2}$ retrieval from near infrared spectra of SCIAMACHY onboard ENVISAT, Atmos. Chem. Phys., 5, 9-22, 2005,

http://www.atmos-chem-phys.net/5/9/2005/.

Langridge, J. M., Stephen, M. B., and Jones, R. L.: A compact broadband cavity enhanced absorption spectrometer for detection of atmospheric $\mathrm{NO}_{2}$ using light emitting diodes, Analyst, 131, 916-922, 2006.

Langridge, J. M., Laurilla, T., Watt, R. S., Jones, R. L., Kaminski, C. F., and Hult, J.: Cavity enhanced absorption spectroscopy of multiple trace gas species using a supercontinuum radiation source, Optics Express, 16, 10178-10188, 2008.

Marquard, L. C., Wagner, T., and Platt, U.: Improved approaches for the calculation of air mass factors required for scattered light differential optical absorption spectroscopy, J. Geophys. Res., 105, 1315-1327, 2000.

Maurellis, A. N., Lang, R., and van der Zande, W. J.: A new DOAS parametrization for retrieval of trace gases with highly-structured absorption spectra, Geophys. Res. Lett., 27, 4069-4072, 2000.

Meinen, J.: Design and Assembling of a Broadband Cavity Ringdown and Cavity Enhanced Absorption Spectrometer using Light Emitting Diodes. Diploma Thesis in Technical Physics, Technical University of Ilmenau, 2007.

Meinen, J., Thieser, J., Platt, U., and Leisner, T.: Using a high finesse optical resonator to provide a long light path for differential optical absorption spectroscopy: CE-DOAS, Atmos. Chem. Phys. Discuss., 8, 10665-10695, 2008, http://www.atmos-chem-phys-discuss.net/8/10665/2008/.

Paldus, B. A. and Zare, R. N.: CRDS an historical perspective and introduction, in: Cavity-Ringdown Spectroscopy: An UltralowAbsorption Measurement Technique, edited by: Busch, K. W. and Busch, M. A., ACS Washington D.C., 1999.

Peeters, R., Berden, G., Apituley, A., and Meijer, G.: Openpath trace gas detection of ammonia based on cavityenhanced absorption spectroscopy, Appl. Phys. B, 71, 231-236, doi:10.1007/s003400000302, 2000.

Platt, U.: Differential optical absorption spectroscopy (DOAS), in: Air Monitoring by Spectroscopic Techniques, edited by: Sigrist, M. W., Chemical Analysis Series, vol. 127, John Wiley \& Sons, Inc., 1994.
Platt, U., Perner, D., and Pätz, H. W.: Simultaneous measurement of atmospheric $\mathrm{CH}_{2} \mathrm{O}, \mathrm{O}_{3}$, and $\mathrm{NO}_{2}$ by differential optical absorption, J. Geophys. Res., 84, 6329-6335, 1979.

Platt, U., Marquard, L., Wagner, T., and Perner, D.: Corrections for zenith scattered light DOAS, Geophys. Res. Lett., 24, 17591762, 1997.

Platt, U. and Stutz, J.: Differential Optical Absorption spectroscopy, Principles and Applications, Springer, XV, 597 p. 272 illus., 29 in color, Physics of Earth and Space Environments, ISBN 978-3540-21193-8, 2008.

Siegman, A. E.: Lasers, University Science Books, Mill Valley, 1986, Chapter 14, pp. 558-579, 1986.

Simpson, W. R.: Continuous wave cavity ring-down spectroscopy applied to in situ detection of dinitrogen pentoxide $\left(\mathrm{N}_{2} \mathrm{O}_{5}\right)$, Rev. Sci. Instr., 74(7), 3442-3452, 2003.

Thieser J.: Cavity Enhanced DOAS: Gerteentwicklung und in-situ Feldmessungen von $\mathrm{NO}_{3}$, Diploma Thesis, University of Heidelberg, 2008.

Thompson, J. E. and Spangler, H. D.: Tungsten source integrated cavity output spectroscopy for the determination of ambient atmospheric extinction coefficient, Appl. Phys. B, 91, 195-201, 2006.

Triki, M., Cermak, P., Méjean, G., and Romanini, D.: Cavityenhanced absorption spectroscopy with a red LED source for NOx trace analysis, Appl. Phys. B, 91, 195-201, 2008.

Venables, D. S., Gherman, T., Orphal, J., Wenger, J. C., and Ruth, A. A.: High Sensitive in Situ Monitoring of $\mathrm{NO}_{3}$ in an Atmospheric Simulation Chamber Using Incoherent Broadband Cavity-Enhanced Absorption Spectroscopy, Environ. Sci. Technol., 40, 6758-6763, 2006.

Volkamer, R., Etzkorn, T., Geyer, A., and Platt, U.: Correction of the oxygen interference with UV spectroscopic (DOAS) measurements of monocyclic aromatic hydrocarbons in the atmosphere, Atmos. Environ., 32, 3731-3747, 1998.

Volkamer, R.: A DOAS Study on the Oxidation Mechanism of Aromatic Hydrocarbons under Simulated Atmospheric Conditions, Doktoral Thesis, University of Heidelberg, Germany, 2001.

Washenfelder, R. A., Langford, A. O., Fuchs, H., and Brown, S. S.: Measurement of glyoxal using an incoherent broadband cavity enhanced absorption spectrometer, Atmos. Chem. Phys., 8, 7779-7793, 2008, http://www.atmos-chem-phys.net/8/7779/2008/.

Wheeler, M. D., Newman, S. M., Orr-Ewing, A. J., and Ashfold, M. N. R.: Cavity ring-down spectroscopy, J. Chem. Soc., Faraday Trans., 94(3), 337-351, 1998.

Zalicki, P. and Zare, R. N.: Cavity ring-down spectroscopy for quantitative absorption measurements, J. Chem. Phys., 102(7), 2708-2717, 1995. 